\title{
MdVQ37 overexpression reduces basal thermotolerance in transgenic apple by affecting transcription factor activity and salicylic acid homeostasis
}

\author{
Qinglong Dong ${ }^{1}$, Dingyue Duan ${ }^{1}$, Wenqian Zheng ${ }^{1}$, Dong Huang ${ }^{1}$, Qian Wang ${ }^{1}$, Xiaoran $\mathrm{Li}^{1}, \mathrm{Ke}$ Mao ${ }^{1 凶}$ and \\ Fengwang $\mathrm{Ma}^{1 凶}$
}

\begin{abstract}
High temperature $(\mathrm{HT})$ is one of the most important environmental stress factors and seriously threatens plant growth, development, and production. VQ motif-containing proteins are transcriptional regulators that have been reported to regulate plant growth and developmental processes, including responses to biotic and abiotic stresses. However, the relationships between VQ motif-containing proteins and HT stress have not been studied in depth in plants. In this study, transgenic apple (Malus domestica) plants overexpressing the apple VQ motif-containing protein-coding gene (MdVQ37) were exposed to HT stress, and the transgenic lines exhibited a heat-sensitive phenotype. In addition, physiological and biochemical studies revealed that, compared with WT plants, transgenic lines had lower enzymatic activity and photosynthetic capacity and lower amounts of nonenzymatic antioxidant system metabolites under HT stress. Transcriptome analysis revealed 1379 genes whose expression differed between the transgenic lines and WT plants. GO and KEGG pathway analyses showed that transcription factor activity and plant hormone signaling pathways were differentially influenced and enriched in the transgenic lines. Salicylic acid (SA) content analysis indicated that overexpression of MdVQ37 reduced the content of endogenous SA by regulating the expression of SA catabolism-related genes, which ultimately resulted in disruption of the SA-dependent signaling pathway under HT stress. The application of SA slightly increased the survival rate of the transgenic lines under HT stress. Taken together, our results indicate that apple MdVQ37 has a regulatory function in basal thermotolerance by modulating the activity of transcription factors and SA homeostasis. Overall, this study provides novel insights that improve our understanding of the various functions of VQ motif-containing proteins.
\end{abstract}

\section{Introduction}

As sessile organisms, plants cannot escape the effects of environmental stress during their growth and development, and high temperature (HT) is among the major types of environmental stress. As global warming

Correspondence: Ke Mao (maoke2002@nwsuaf.edu.cn) or

Fengwang Ma (fwm64@nwsuaf.edu.cn)

State Key Laboratory of Crop Stress Biology for Arid Areas/Shaanxi Key

Laboratory of Apple, College of Horticulture, Northwest A \& F University,

712100 Yangling, Shaanxi, China

These authors contributed equally: Qinglong Dong, Dingyue Duan intensifies, the frequency and extent of extreme HTs continue to increase, posing a major threat to plant growth and development, crop yields, and food security ${ }^{1,2}$. Plants exhibit a heat shock response under HT stress. They also have the ability to survive direct exposure to extreme HT (i.e., basal thermotolerance) and can also increase their tolerance to lethal HT stress after acclimation to nonlethal HT stress for a certain period of time (i.e., acquired thermotolerance) ${ }^{3-5}$.

After plants are subjected to HT stress, a series of damaging events occur. First, HT stress causes severe

\section{(c) The Author(s) 2021}

(c) (i) Open Access This article is licensed under a Creative Commons Attribution 4.0 International License, which permits use, sharing, adaptation, distribution and reproduction cc) in any medium or format, as long as you give appropriate credit to the original author(s) and the source, provide a link to the Creative Commons license, and indicate if changes were made. The images or other third party material in this article are included in the article's Creative Commons license, unless indicated otherwise in a credit line to the material. If material is not included in the article's Creative Commons license and your intended use is not permitted by statutory regulation or exceeds the permitted use, you will need to obtain permission directly from the copyright holder. To view a copy of this license, visit http://creativecommons.org/licenses/by/4.0/. 
damage to thylakoids, severely limiting the photosynthetic electron transfer rate of plant photosystems (PSs) and the activity of several related enzymes and rapidly inhibiting photosynthesis in plants ${ }^{6}$. Second, HT stress disrupts the oxygen-evolving complex, resulting in an imbalance during the energy conversion between the photosystem II (PSII) oxygen-evolving complex and the receptor site of the photosystem, resulting in irreversible damage to PSII and greatly reducing its activity ${ }^{6,7}$. HT can also cause plant stomata to close and affects leaf stomatal conductance (gs) and intercellular $\mathrm{CO}_{2}$ concentrations ${ }^{8,9}$. Furthermore, HT stress can disrupt the dynamic balance between harmful reactive oxygen species (ROS) production and removal in plants, and the accumulation of superoxide anions $\left(\mathrm{O}_{2} .-\right)$, hydrogen peroxide $\left(\mathrm{H}_{2} \mathrm{O}_{2}\right)$, singlet oxygen species $\left({ }^{1} \mathrm{O}_{2}\right)$, and hydroxyl radicals $(\mathrm{OH}-)$ increases the degree of membrane lipid peroxidation, ultimately leading to oxidative stress ${ }^{6,10}$.

Plants have evolved complex and diverse strategies to accommodate HT stress. Initially, plants evolved antioxidant defense systems under HT stress to reduce the harmful effects of oxidative stress ${ }^{6}$. Plant antioxidant defense systems include nonenzymatic and enzymatic ROS-scavenging systems. The nonenzymatic ROS-scavenging system consists mainly of carotenoids, ascorbic acid (AsA), and glutathione (GSH), whereas the enzymatic system mainly includes peroxidase (POD), catalase (CAT), superoxide dismutase (SOD), glutathione reductase (GR), ascorbate peroxidase (APX), dehydroascorbate reductase (DHAR) and monodehydroascorbate reductase (MDHAR) $)^{6,11}$. Furthermore, under HT stress, plants can accumulate osmotic adjustment substances to maintain plant cell osmotic pressure and reduce stress-induced damage. Osmotic adjustment substances include proline, soluble sugars, soluble protein, betaine, and polyamines ${ }^{6}$. In addition, when plants are subjected to HT stress, their response at the molecular level is to synthesize a variety of heat-shock proteins and heat stress-responsive transcription factors (TFs), thereby enhancing the ability of plants to resist $\mathrm{HT}$ stress ${ }^{10}$. Heat stress-responsive TFs include HSF, AP2/ERF, bZIP, MYB/ MYC, NAC (NAM, ATAF, and CUC), and WRKY TFs ${ }^{12-16}$.

Several studies have indicated that salicylic acid (SA) is an important phenolic compound that acts as an endogenous plant signaling molecule and is involved in the regulation of plant growth, development and defense responses ${ }^{17}$. The function of $\mathrm{SA}$ in plant-pathogen interactions has been well documented. In addition to biotic stress, SA participates in the activation of several plants abiotic responses ${ }^{17-20}$. For example, SA can help counter the adverse effects of heat and salt stress on photosynthesis, AsA-GSH, and the activities of ROSscavenging enzymes in wheat and choy sum, respectively $^{18,20}$. Overexpression of LCSABP2, which is the SAbinding protein 2-encoding gene from Lycium chinense, in transgenic tobacco lines resulted in lower ROS levels, higher endogenous SA contents, and photosynthetic capacities, and improved tolerance to poor nutrient stress $^{21}$.

In 2002, the first VQ motif-containing protein (SIGMA FACTOR-BINDING PROTEIN 1, VQ23/SIB1) was discovered in Arabidopsis $^{22}$. A series of subsequent studies revealed that VQ motif-containing proteins participate not only in the regulation of plant growth but also in developmental processes ${ }^{23}$. For example, the $v q 14 / i k u 1$ mutant exhibits a small-seed phenotype, and VQ18 and VQ26 were responsive to abscisic acid (ABA) treatment, indicating that these VQ motif-containing proteins regulate seed size and germination ${ }^{24,25}$. The $v q 29$ loss-offunction mutant displays decreased hypocotyl elongation under white light and low-intensity far-red light ${ }^{26}$. These results suggest that VQ29 negatively regulates seedling photomorphogenesis ${ }^{26}$. The triple-mutant wrky2-1 wrky34-1 vq20-1 presents compromised pollen germination, development, and tube growth, suggesting that VQ20 affects plant male gametogenesis ${ }^{27}$. In addition, VQ motif-containing proteins have been found to participate in the plant defense response and stress tolerance. For instance, amiR-vq12 vq29 double-mutants present significantly enhanced disease resistance to the fungus Botrytis cinerea, whereas overexpression of $V Q 12$ or $V Q 29$ increased susceptibility to $B$. cinerea $^{28}$. These results suggested that VQ12 and VQ29 play negative regulatory roles in the response to $B$. cinerea infection by functioning as negative regulators of plant basal defense against $B$. cinerea. Compared with wild-type (WT) plants, mutant $v q 9$ plants show increased tolerance to salt stress, whereas the overexpression of $V Q 9$ renders plants hypersensitive to salt stress ${ }^{29}$. These results indicated that VQ9 functions as a negative regulator of the response to salt stress. To date, few studies have functionally analyzed the biological functions of VQ motif-containing proteins in the HT stress response ${ }^{30}$.

Apple (Malus domestica) trees are one of the most important fruit tree species in temperate regions of the world and tolerate a wide range of cultivation areas. However, HT stress severely reduces apple growth, development, quality, and production. Here, we examined the expression patterns of various apple $M d V Q$ genes and found that $M d V Q 37$ was clearly downregulated in response to $\mathrm{HT}$ stress. In addition, $M d V Q 37$-overexpressing apple plants were used to elucidate the biological function of this gene in the HT stress response. We found that $M d V Q 37$ overexpression reduced basal thermotolerance in transgenic apple lines. This negative response was related to a production of greater amounts of toxic ROS, a reduction in the AsA-GSH level, and interference with photosynthesis compared with those in WT plants. Additional analysis suggested that this 
negative effect stemmed from changes in TF activity and SA accumulation levels. Generally, these findings provide novel insights that improve our understanding of the multiple functions of VQ motif-containing proteins. At the same time, these findings may have important applications in the breeding of horticultural plants in the face of the frequent occurrence of extreme climate conditions.

\section{Results}

Analysis of the expression of MdVQ genes under HT stress

In a previous study, we found and identified VQ family members in the apple genome and thoroughly analyzed the evolution and structure of apple $M d V Q$ genes, as well as the biological functions of some MdVQ members ${ }^{31}$. In this study, we analyzed the expression levels of various $M d V Q$ genes under HT stress via RT-qPCR. The expression levels of $M d V Q 1, M d V Q 2, M d V Q 12$, and $M d V Q 25$ were significantly upregulated in response to HT stress, whereas those of $M d V Q 4, M d V Q 6, M d V Q 16$, MdVQ20, MdVQ26, MdVQ31, MdVQ34, MdVQ37, $M d V Q 38, M d V Q 42$, and $M d V Q 44$ were significantly downregulated (Fig. S1). Among these genes, the expression of $M d V Q 12$ increased 8.45-fold after treatment for $4 \mathrm{~h}$ compared with that of the control (Fig. S1); the expression level of $M d V Q 37$ decreased 49-fold after treatment for $8 \mathrm{~h}$ compared with that of the control (Fig. S1). Taken together, these results suggest that multiple VQ genes are involved in the response to HT stress in apples.

\section{Overexpression of MdVQ37 increased sensitivity to HT stress in apple}

Based on the expression patterns of $M d V Q$ genes under HT stress (Fig. S1) and the screening results of interactions between MdVQ motif-containing proteins and MdWRKY transcription factors ${ }^{31}$, three $M d V Q$ genes, $M d V Q 6, M d V Q 34$, and $M d V Q 37$, were selected for subsequent genetic transformation and functional identification in apple. Due to the extremely low efficiency of transgene expression in apple plants, only two $M d V Q 37-$ overexpressing lines (L1 and L2) were obtained, and no lines with significantly reduced $M d V Q 37$ expression were obtained via genetic transformation. Compared with those in WT plants, the transcript levels of $M d V Q 37$ in L1 and L2 constitutively increased by 124.88 -fold and 85.36fold, respectively (Fig. S2). After exposure to $48^{\circ} \mathrm{C}$ for $6 \mathrm{~h}$, the top leaves of the transgenic lines wilted severely and displayed necrotic spots. In contrast, the top leaves of WT plants showed symptoms of only slight dehydration, and the fully expanded leaves of the lower parts of the plants remained green and vigorous (Fig. 1A). Electrolyte leakage (EL) and malondialdehyde (MDA) levels were used as indicators of abiotic stress-induced membrane damage $^{9,11}$. Thus, we measured the levels of EL in the WT plants and transgenic lines under HT stress. The levels of EL increased significantly due to the effect of HT stress, and the EL levels in the WT plants were lower than those in the transgenic lines (Fig. 1B). Similar trends in the MDA content were observed in the WT plants and transgenic lines under HT stress (Fig. 1C), which indicated that the transgenic lines sustained substantially more damage from HT stress. In addition, although HT stress caused an increase in proline content in the leaves, we found that the proline content in the transgenic lines was significantly lower than that in the WT plants (Fig. 1D). Following exposure to HT, the total chlorophyll concentration of the WT plants did not change, but the total chlorophyll concentration of the transgenic lines significantly decreased (Fig. 1E). To further evaluate the $\mathrm{HT}$ stress tolerance of the $M d V Q 37$ transgenic lines, we subjected the WT plants and transgenic lines to $48^{\circ} \mathrm{C}$ for $24 \mathrm{~h}$ (Fig. S3). After the plants were allowed to recover at $24^{\circ} \mathrm{C}$ for $48 \mathrm{~h}$, we determined the survival rate of the WT plants and transgenic lines. The results in Fig. 1F reveal that the survival rate of the transgenic lines and WT plants was significantly reduced, but the survival rate of the WT plants was much higher than that of the transgenic lines. All of these findings indicated that the transgenic lines sustained increased physiological damage under $\mathrm{HT}$ stress, implying that $M d V Q 37$ plays a negative role in the response to HT stress in apples.

\section{Overexpression of MdVQ37 increased ROS accumulation and reduced antioxidant enzyme activities under heat stress}

The excessive accumulation of ROS such as $\mathrm{O}_{2} \cdot-$ and $\mathrm{H}_{2} \mathrm{O}_{2}$ can lead to oxidative stress, damage macromolecules, and cell membrane lipid structures, and cause harmful effects in plants ${ }^{32}$. For this reason, we investigated the effects of the overexpression of $M d V Q 37$ by measuring the in situ accumulation of $\mathrm{O}_{2} \cdot-$ and $\mathrm{H}_{2} \mathrm{O}_{2}$ via nitro blue tetrazolium (NBT) and 3,3'-diaminobenzidine (DAB) histochemical staining, respectively. The leaves of the transgenic lines showed stronger brown or blue spots after HT stress than did the leaves of the WT plants (Fig. 2A, B). This result implies that the transgenic lines accumulated more ROS than the WT plants did under HT stress. The quantitative measurements of $\mathrm{O}_{2} \cdot-$ and $\mathrm{H}_{2} \mathrm{O}_{2}$ were consistent with the above data (Fig. 2C, D). SOD, CAT, and POD are three effective antioxidant enzymes involved in ROS scavenging ${ }^{33}$. Therefore, the activities of SOD, CAT, and POD enzymes were measured in the transgenic lines and WT plants. The activities of the CAT and SOD enzymes in WT plants were significantly higher than those in transgenic lines under normal conditions (Fig. 2E-G). After $6 \mathrm{~h}$ of heat exposure, the activities of these three enzymes increased significantly in the apple plants. However, this increase was 

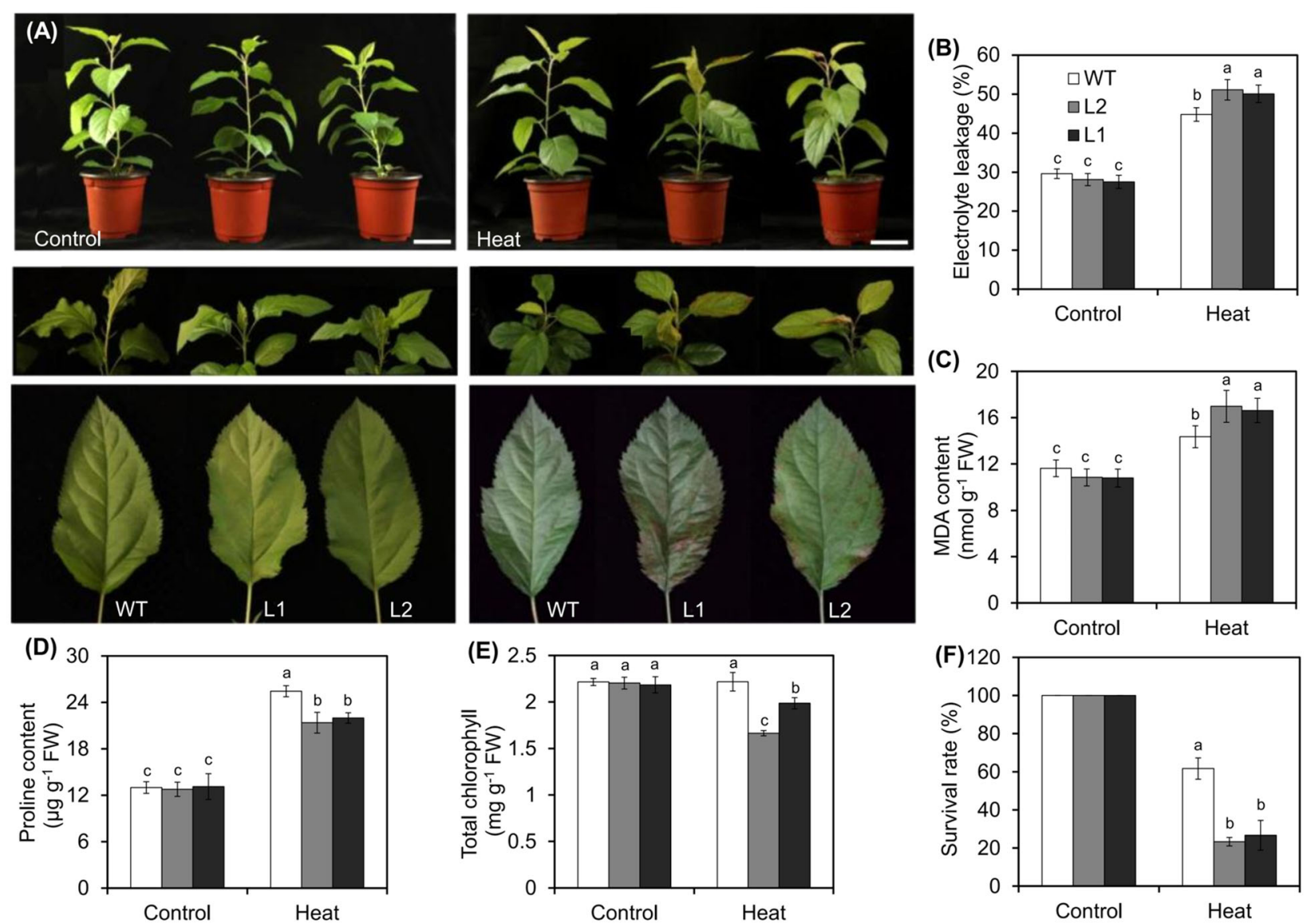

Fig. 1 Overexpression of MdVQ37 increases sensitivity to HT stress in transgenic apple lines. A Phenotypes of WT plants and MdVQ37 transgenic lines under HT stress. Ninety-day-old WT plants and transgenic lines of uniform size were exposed to HT stress for $6 \mathrm{~h}$ in red plastic pots $(10 \times 10 \times 8.5 \mathrm{~cm})$. The plants were imaged after 0 and $6 \mathrm{~h}$ of HT stress. B Electrolyte leakage (EL), (C) malondialdehyde (MDA) content, (D) proline content, $(\mathbf{E})$ chlorophyll concentration, and (F) survival rate of WT plants and MdVQ37 transgenic lines subjected to HT stress or not. The data are presented as the means \pm SEs of three biological experiments. The different letters represent significant differences among the treatments on each date (one-way analysis of variance (ANOVA) followed by Duncan's test, $p<0.05$ )

significantly lower in the transgenic lines compared with the WT plants (Fig. 2E-G). Overall, our data indicated that overexpression of $M d V Q 37$ reduced antioxidant enzyme activities, thereby resulting in excessive ROS production under HT stress.

\section{Overexpression of MdVQ37 reduced the efficiency of the AsA-GSH cycle under HT stress}

It is known that the AsA-GSH cycle is an important antioxidant defense system that works against oxidative damage in plants ${ }^{9,34}$. Therefore, to further study the effect of MdVQ37 on the AsA-GSH cycle under HT stress, we determined the concentrations of AsA and GSH in the transgenic lines and WT plants. Under normal conditions, the total ascorbate (AsA + dehydroascorbate (DHA)) content, total glutathione (GSH + glutathione disulfide (GSSG)) content, and the ratios of AsA/DHA and GSH/ GSSG were at the same levels in the transgenic lines and
WT plants (Fig. 3A-H). However, after $6 \mathrm{~h}$ of exposure to HT stress, the contents of AsA, DHA, and AsA + DHA in the WT plants and transgenic lines significantly increased, although the contents of AsA and AsA + DHA in the transgenic lines were significantly lower than those in the WT plants (Fig. 3A, C). In addition, the DHA content in the transgenic lines was significantly higher than that in the WT plants (Fig. 3B), and the AsA/DHA ratio in the transgenic lines decreased more markedly than it did in the WT plants under HT stress (Fig. 3D). The levels of GSH, GSSG, and GSH + GSSG and the GSH/GSSG ratio exhibited similar trends (Fig. 3E-H). Moreover, similar to the changes in the contents and trends of AsA and GSH, the measurements of the activities of the APX, GR, DHAR, and MDHAR enzymes involved in the AsA-GSH cycle in the transgenic lines and WT plants revealed that after $6 \mathrm{~h}$ of exposure to HT stress, the activities were significantly reduced in the transgenic lines compared 


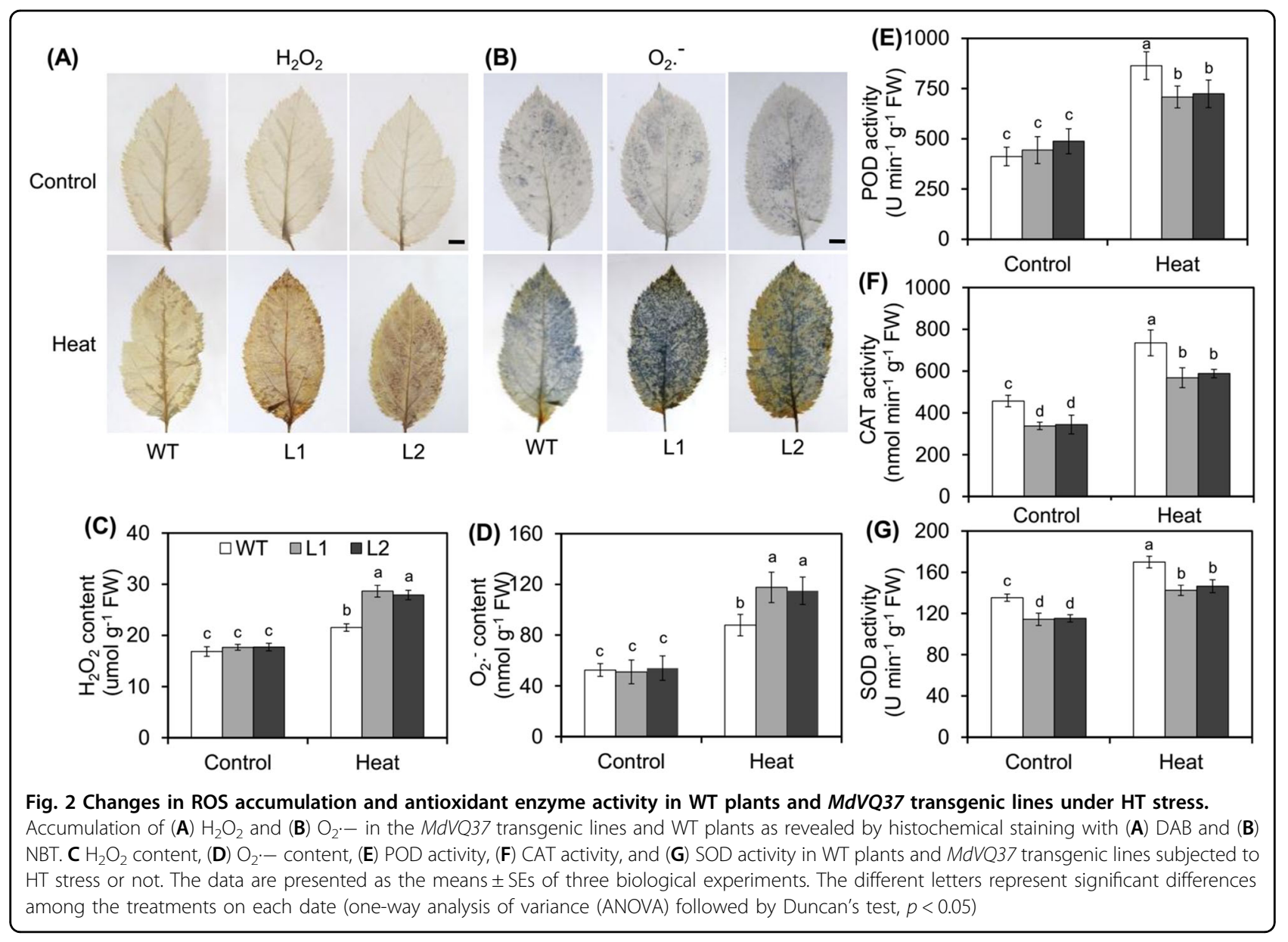

with the WT plants (Fig. 3I-L). Taken together, these results revealed that the overexpression of $M d V Q 37$ in transgenic apple plants attenuated the antioxidant capacity of AsA-GSH recycling under HT stress.

\section{Overexpression of MdVQ37 aggravated the photoinhibition of the photosynthetic system under HT stress}

HT stress causes leaf stomata to close, thereby reducing photosynthetic efficiency and affecting plant growth. Therefore, we compared the stomatal morphology between WT plants and transgenic lines under HT stress. No difference in the stomatal opening was observed between the WT plants and transgenic lines under normal-temperature conditions (Fig. 4A, B). However, after $4 \mathrm{~h}$ of heat exposure, the stomatal shrinkage of the transgenic lines increased compared with that of the WT plants (Fig. 4A, B). Furthermore, a comparison of the measurements of photosynthesis-related parameters between the transgenic lines and WT plants under HT stress revealed, that under normal conditions, there were no differences in net photosynthesis $(\mathrm{Pn})$, stomatal conductance $\left(\mathrm{g}_{\mathrm{s}}\right)$, intercellular $\mathrm{CO}_{2}$ concentration $(\mathrm{Ci})$, or transpiration rate $(\mathrm{Tr})$ between the WT plants and transgenic lines (Fig. 4C-F). After $6 \mathrm{~h}$ of exposure to HT stress followed by recovery for $24 \mathrm{~h}$, the Pn value decreased sharply. The Pn value of the WT plants was approximately $29.9 \%$ that of the controls, whereas that of the transgenic lines was approximately 19.5 and $22.6 \%$ that of those of the controls (Fig. 4C). Consistent with the Pn data, the $g_{s}$ and $\operatorname{Tr}$ data showed similar trends after the recovery period (Fig. 4D, F). However, the change in the $\mathrm{Ci}$ value between the WT plants and transgenic lines during the recovery period was small (Fig. 4E). Taken together, the above results indicate that the photosynthetic function of $M d V Q 37$ was more compromised in the transgenic lines than in the WT plants under HT stress.

Analysis of the maximum photochemical efficiency of photosystem II (PSII) photochemistry (variable fluorescence/maximum fluorescence $(\mathrm{Fv} / \mathrm{Fm}))$, a valuable criterion for studying the effect of abiotic stress on plant photosynthesis $^{35,36}$, via fluorescence imaging showed that transgenic lines and WT plants initially exhibited similar fluorescence intensities under normal conditions. However, the fluorescence of the transgenic lines became more intense than that of the WT plants after $24 \mathrm{~h}$ of recovery 

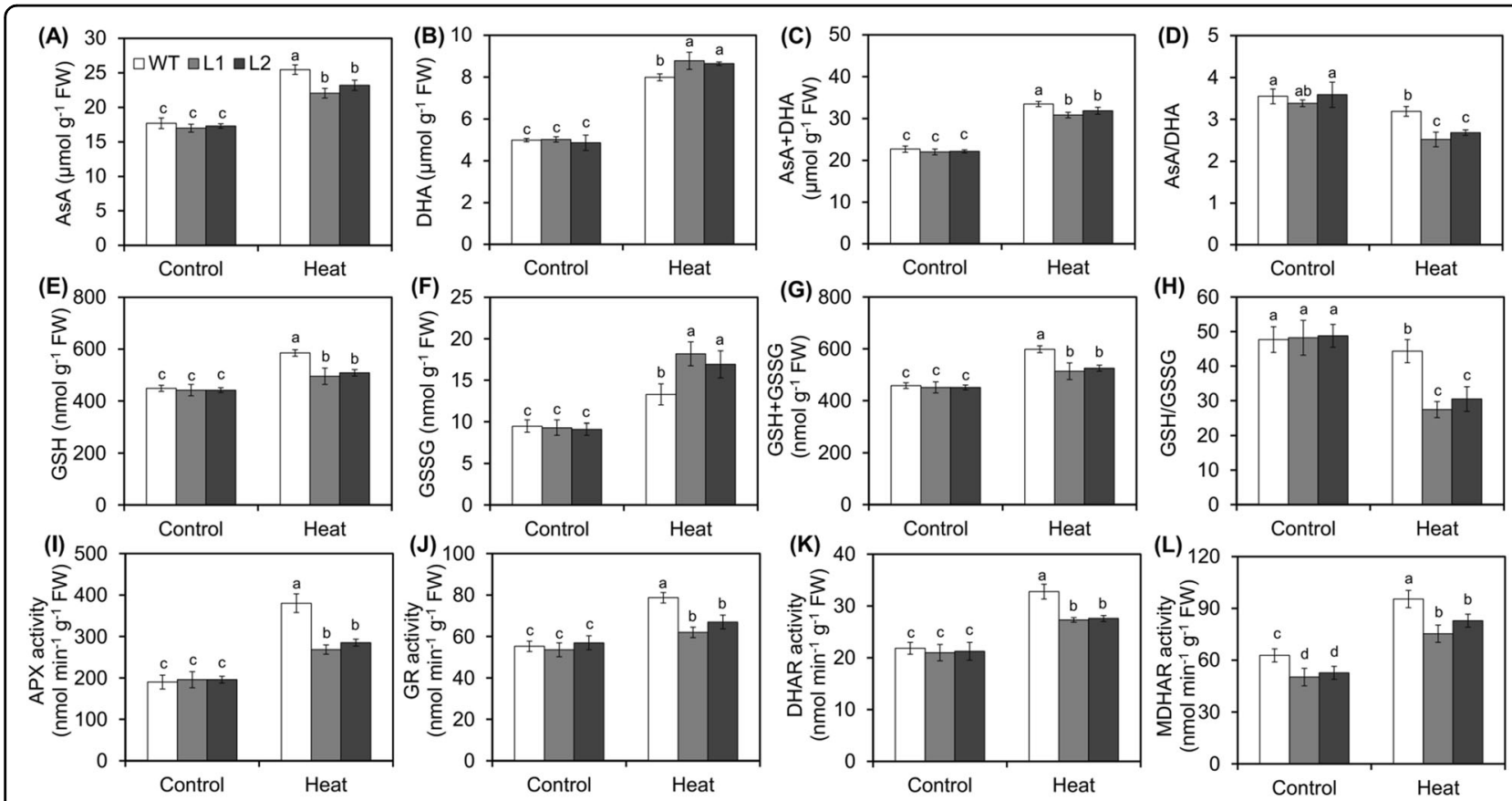

Fig. 3 Changes in antioxidant contents and activities of the main antioxidative enzymes in the ascorbate-glutathione (AsA-GSH) cycle under HT stress. A AsA content, (B) DHA content, (C) AsA + DHA content, (D) AsA/DHA content, (E) GSH content, (F) GSSG content, (G) GSH + GSSG content, (H) GSH/GSSG, (I) APX activity, (J) GR activity, (K) DHAR activity, and (L) MDHAR activity in WT plants and MdVQ37 transgenic lines subjected to HT stress or not. The data are presented as the means \pm SEs of three biological experiments. The different letters represent significant differences among the treatments on each date (one-way analysis of variance (ANOVA) followed by Duncan's test, $p<0.05$ )

from HT stress (Fig. 5A). Consistent with the fluorescence imaging results, the $\mathrm{Fv} / \mathrm{Fm}$ ratio, a parameter representing the health and growth of plants, of the transgenic lines was equivalent to that of the WT plants under control conditions but was significantly lower after $24 \mathrm{~h}$ of recovery from HT stress (Fig. 5B). Further analysis of the photochemical changes in PSI and PSII using a DualPAM-100 measurement system to evaluate the energy changes in PSI and $\mathrm{PSII}^{37}$ revealed that, initially, the transgenic and WT plants exhibited similar Y(I), ETR(I), $\mathrm{Y}(\mathrm{II})$, and ETR(II) values under normal conditions. However, these indicators significantly decreased after $24 \mathrm{~h}$ of recovery from HT stress (Fig. 5C-F). Specifically, the $\mathrm{Y}(\mathrm{II})$ values of line 1 and line 2 were 0.64 and 0.79 times that of WT plants under HT stress, respectively (Fig. 5D). Similar trends were also found in the changes in ETR (I), ETR (II), and Y (I) under HT stress (Fig. 5C, E, F). Overall, these results revealed that overexpression of $M d V Q 37$ in the transgenic plants had negative effects on photochemical reactions, including those of both PSI and PSII, under HT-stress conditions.

\section{MdVQ37 overexpression affects TF activity and plant hormone signaling}

The transcriptional differences between WT plants and $M d V Q 37$ transgenic lines were further characterized via transcriptome analysis (RNA-seq) of transgenic lines (37-1 and 37-2) and WT plants under normal conditions. After filtering, approximately 43.9 million clean reads were obtained for each genotype, with a total mapping percentage of $93.3 \%$ (Table S1). There were 1,379 differentially expressed genes (DEGs) $(\mid \log 2$ (fold change) $\mid \geq 1$, padj $\leq 0.05$ ), of which 837 were downregulated and 542 were upregulated in the $37-1$ vs. WT and $37-2$ vs. WT comparison groups, respectively (Fig. 6A; Table S2). To verify the accuracy of the RNA-seq data, 14 DEGs, including five upregulated and nine downregulated genes, were selected for transcript level measurements via qRTPCR analysis (Fig. S4). The results of the qRT-PCR analysis of the expression of the 14 selected genes were consistent with transcript abundance as revealed by the RNA-seq data, suggesting that the DEG screening based on the RNA-seq analysis was reliable. Furthermore, we analyzed the expression patterns of these 14 DEGs under HT stress. Compared with WT plants, MdVQ37 transgenic plants presented significantly lower transcript levels of MdHSFA3, MdWRKY33, MdNAC2, MdMYB6, $M d R A V 1, M d P O D, M d M D H A R$, and $M d C A T$ and increased transcript levels of MdbHLH63 and MdNAC87 under HT stress (Fig. S4). In particular, two genes involved in the ROS regulatory system, MdCAT and $M d M D H A R$, and genes involved in the AsA-GSH cycle 


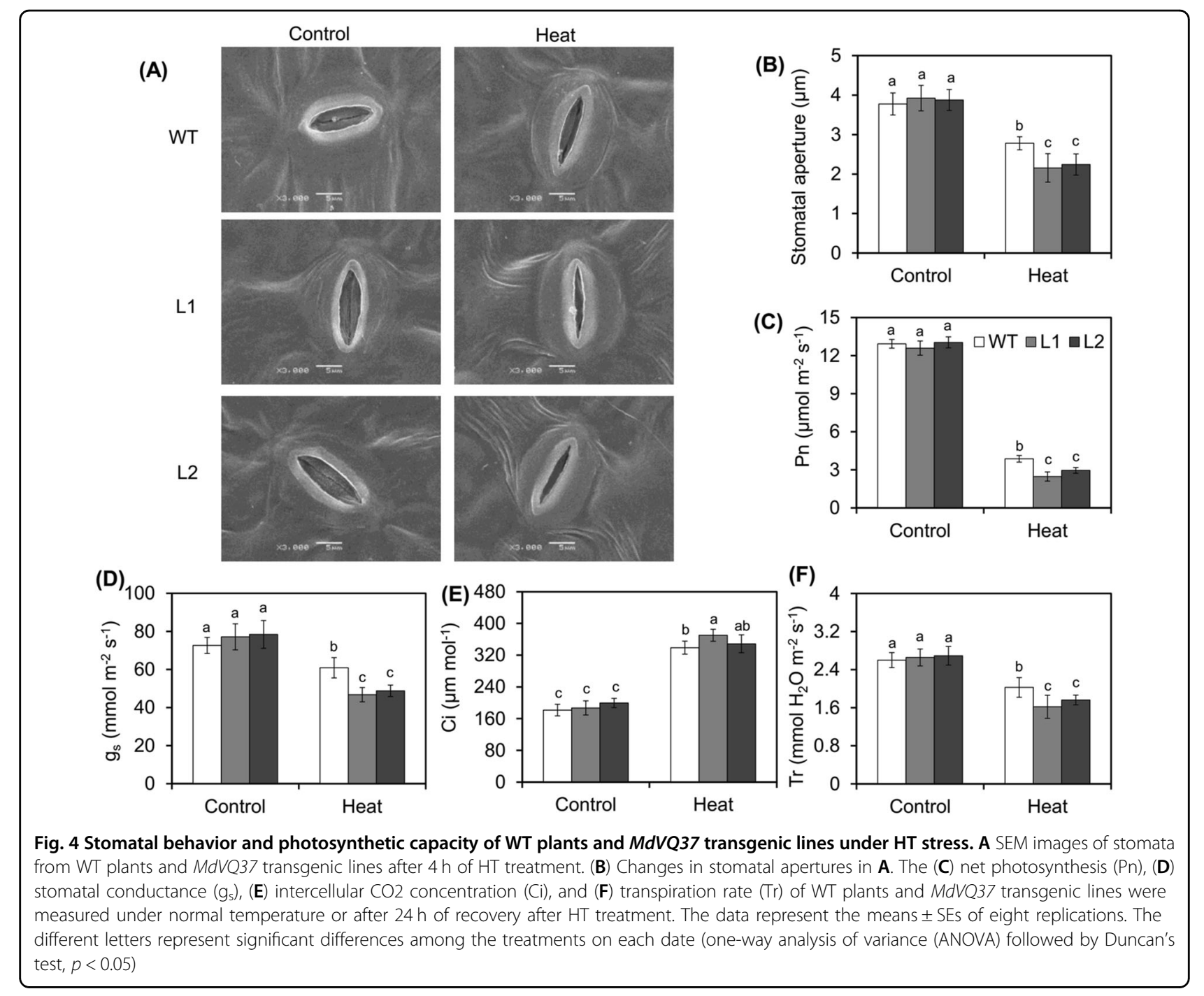

were downregulated in transgenic plants, which was consistent with the measured enzyme activity of CAT and MDHAR under normal conditions and HT stress.

The identified DEGs in the 37-1 vs. WT and 37-2 vs. WT comparison groups were enriched in three major categories, namely, biological processes, molecular functions, and cellular components, which revealed transcriptional differences between the transgenic lines and WT plants. The enriched Gene Ontology (GO) terms in the biological process and cellular component categories were not similar in the 37-1 vs. WT and 37-2 vs. WT groups (Fig. 6B; Table S3). These groups shared seven enriched GO terms for the molecular function category: 'transcription factor activity', 'nucleic acid-binding transcription factor activity', 'sequence-specific DNA binding', 'sequence-specific DNA binding', 'heme binding', 'tetrapyrrole binding' and 'iron ion binding' (Fig. 6B). The top three GO terms, namely, 'transcription factor activity', 'nucleic-acid binding transcription factor activity' and 'sequence-specific DNA binding', were associated with DEGs that encoded various members of the TFs WRKYs, AP2/ERFs, MYBs, NACs, bZIPs, bHLHs, and HSFs (Table S3), which are known to regulate the stress response.

Kyoto Encyclopedia of Genes and Genomes (KEGG) pathway analysis of the DEGs from the $37-1$ vs. WT and 37-2 vs. WT groups led to the identification of 20 major enriched pathways. The most highly enriched KEGG pathway was 'plant hormone signal transduction', followed by 'alpha-linolenic acid metabolism' (Fig. 6C; Table S4).

\section{MdVQ37 overexpression leads to decreases in SA content} and SA-dependent signaling pathways under heat stress

As the analysis of the KEGG results revealed that the transgenic lines induced plant hormone signal transduction (Fig. 6C) and increased transcript levels of genes involved in SA signaling (Table S2), we measured the contents of endogenous SA in the WT plants and 

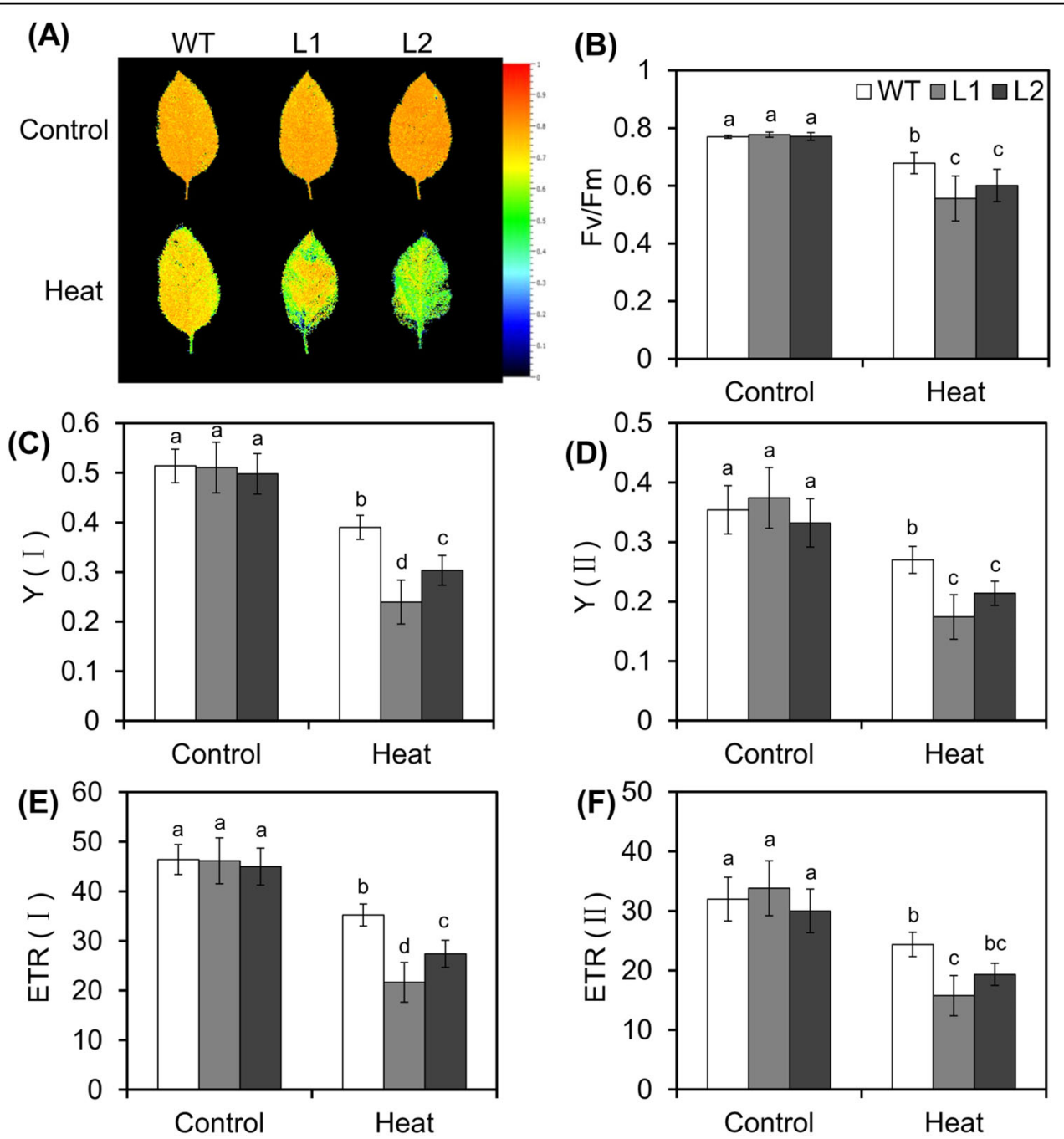

Fig. 5 Capacity of PSI and PSII in WT plants and MdVQ37 transgenic lines after HT treatment. A Chlorophyll fluorescence images (Fv/Fm) and (B) Fv/Fm ratios of MdVQ37 transgenic lines and WT plants under normal temperature or after $24 \mathrm{~h}$ of recovery after $\mathrm{HT}$ treatment. The effective quantum yield and electron transport rate of PSI [Y(I) (C) and ETR (I) (D)] and PSII [Y(II) (E) and ETR (II) (F)] were measured at a normal temperature or after $12 \mathrm{~h}$ of recovery after high-temperature treatment. The data represent the means \pm SEs of eight replications. The different letters represent significant differences among the treatments on each date (one-way analysis of variance (ANOVA) followed by Duncan's test, $p<0.05$ )

transgenic lines. The contents of endogenous free SA and total SA were higher in the WT plants than in the transgenic lines under normal-temperature conditions, suggesting that MdVQ37 affected endogenous SA homeostasis (Fig. 7A, B). After $4 \mathrm{~h}$ of HT stress, however, the free SA content drastically increased in both the WT plants and the transgenic lines, but the increase in the free SA content in the two transgenic lines was significantly lower than that in the WT plants, the levels of which were only 70.3 and $74.6 \%$ that of the WT plants (Fig. 7A). The total SA content exhibited a similar trend in the WT plants but not in the transgenic lines (Fig. 7B). To further understand why the endogenous SA content was reduced in the transgenic lines, we examined changes in the expression of SA biosynthesis-related and catabolism- related genes in the WT plants and transgenic lines. No difference in the transcript levels of SA biosynthesis genes was observed between plants of the different genotypes under normal-temperature conditions or HT stress (Fig. $7 \mathrm{C})$. However, the expression of the SA modificationrelated and catabolism-related genes $M d S 5 H 1$ and $\mathrm{MdS5H} 2$ in the transgenic lines was significantly higher than that in the WT plants (Fig. 7C). MdS5H1 and MdS5H2 encode salicylic acid 5-hydroxylase (S5H), also called 2-oxoglutarate-Fe(II) oxygenase, which catalyzes the conversion of SA into 2,5-dihydroxybenzoic acid (2,5DHBA $)^{38}$. Accordingly, we measured the content of 2,5DHBA in the transgenic lines and WT plants. In contrast to the trend of free SA and total SA contents, the content of 2,5-DHBA in the transgenic lines was higher than that 


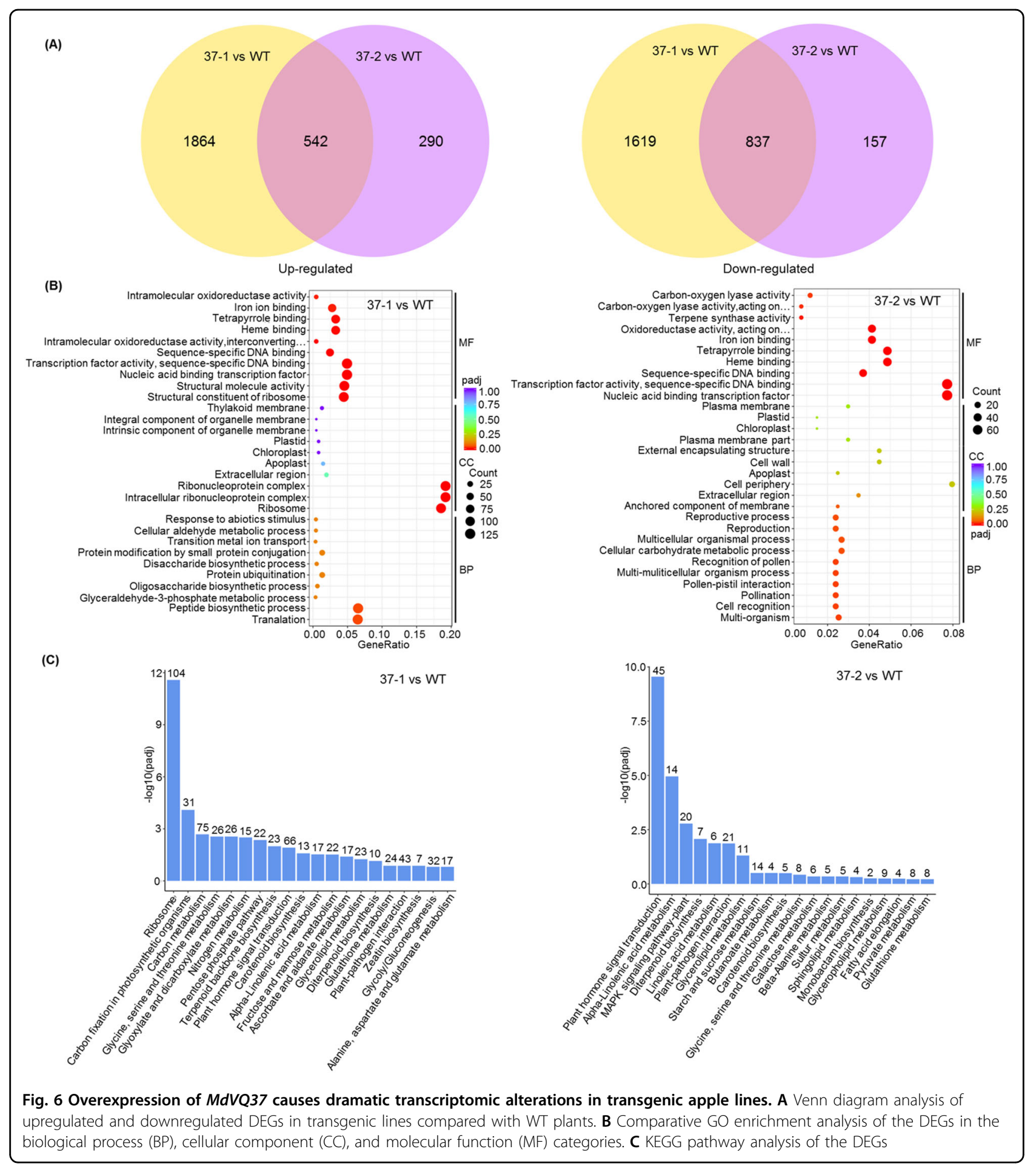

in the WT plants regardless of whether it was measured under normal-temperature conditions or HT stress (Fig. 7D). Furthermore, we measured the expression of SA signaling-related genes in the transgenic lines and WT plants. The expression of most of the SA signaling-related genes in the transgenic lines was lower than that in the WT plants under normal-temperature conditions
(Fig. 7E). Among these genes, MdPAD4, MdPR3, and $M d P R 5$ were induced in response to heat stress, and the transcript levels of these three genes in the transgenic lines were significantly lower than those in the WT plants under HT stress (Fig. 7E), suggesting that MdPAD4, $M d P R 3$, and MdPR5 may play key roles in the resistance of apple to HT stress. 


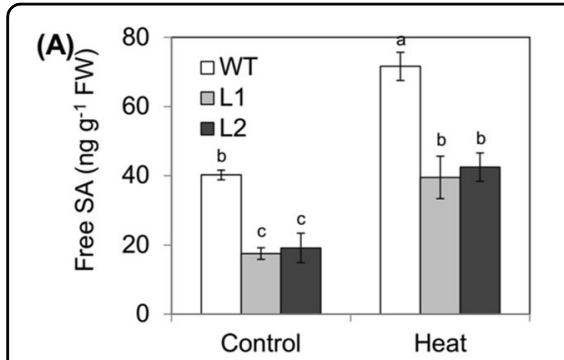

(B)

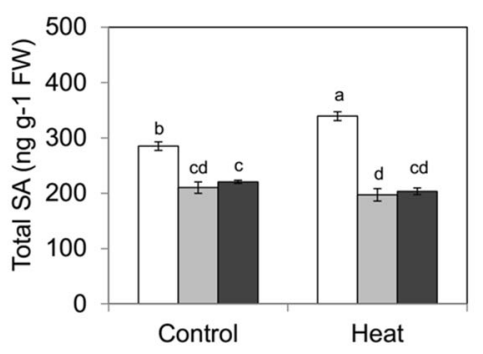

(D)

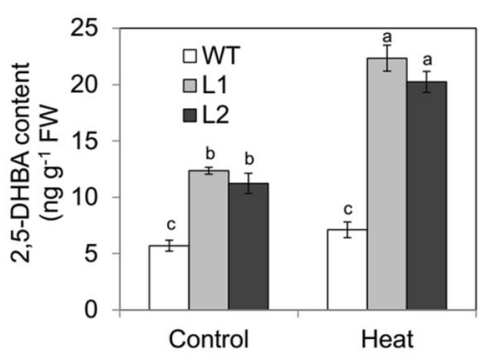

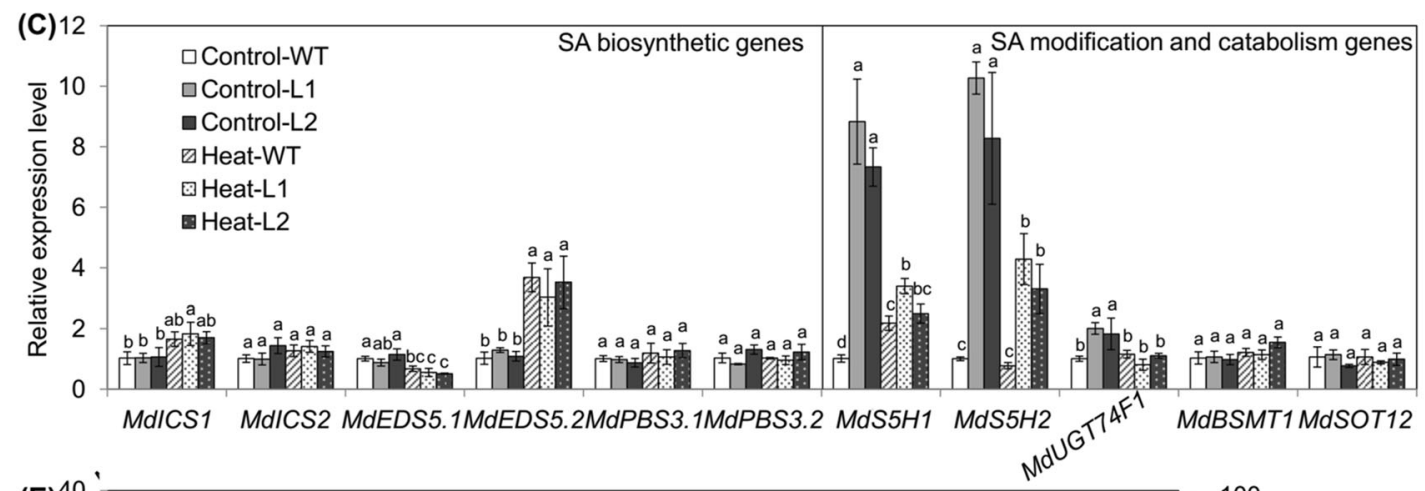
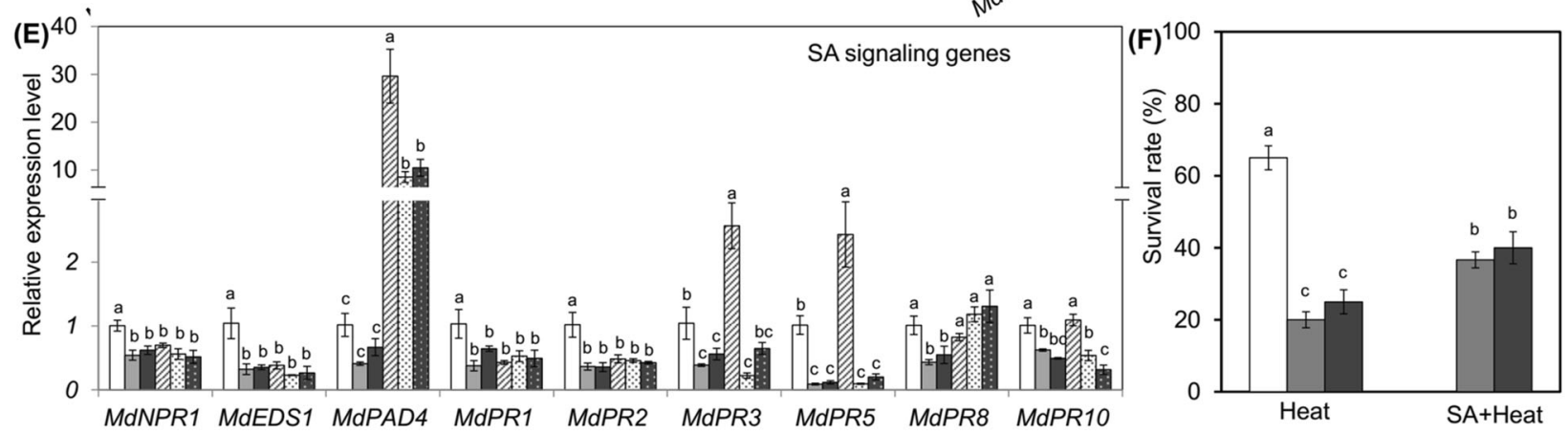

Fig. 7 Effects of heat stress on endogenous SA levels, 2,5-DHBA contents, transcript levels of SA metabolism-related and signaling-related genes, and survival rates of WT plants and MdVQ37 transgenic lines. The contents of endogenous free SA (A) and total SA (B) in WT plants and transgenic lines are subjected to HT stress or not. C Relative transcript levels of SA biosynthesis-related and catabolism-related genes under HT stress. D 2,5-DHBA content in WT plants and transgenic lines subjected to HT stress or not. E Relative transcript levels of SA signaling-related genes under HT stress. F The survival rate of transgenic lines sprayed with $100 \mu \mathrm{M}$ SA under HT stress. The data represent the means \pm SEs of three biological replications. The different letters represent significant differences among the treatments on each date (one-way ANOVA followed by Duncan's test, $p<0.05)$

To determine whether suppression of the SA level in the transgenic lines was responsible for the impaired basic thermotolerance, the transgenic lines were pretreated with $100 \mu \mathrm{M}$ SA. Although the survival rate increased, it was still lower than that of the WT plants (Fig. 7F). Therefore, the reduced basal thermotolerance of the transgenic plants stemmed in part from the decrease in SA levels.

\section{Discussion}

Higher plants have different types of TFs that bind to cis-acting elements in the promoter regions of downstream genes and initiate their transcription ${ }^{23,39}$. VQ motif-containing proteins act as transcriptional regulators controlling a series of biological processes, such as plant growth, development, defense responses, and stress tolerance mechanisms. In model plant species, the biological functions of VQ motif-containing proteins in the response to abiotic stress have been less studied than their functions in the response to biotic stress have, especially HT stress. In fact, the link between VQ motif-containing proteins and basal thermotolerance has not yet been established in plants. Research on the functions of VQ motif-containing proteins in response to HT stress in plants aims to enhance our understanding of the various biological roles of VQ motif-containing proteins. In this study, we used transgenic apple plants overexpressing MdVQ37 to investigate the specific role of MdVQ37 in the response to HT stress. Our results clearly showed that MdVQ37 overexpression reduced tolerance to $\mathrm{HT}$ in 
transgenic apple lines (Fig. 1). HT stress resulted in more damage to $M d V Q 37$ transgenic apple lines than to WT plants through mechanisms involving increased EL, MDA, and ROS levels and decreased proline content, chlorophyll content, and photosynthesis activity (Figs. 1-5).

The accumulation of excessive amounts of ROS is a very important factor contributing to the many adverse effects of HT stress. Previous studies have shown that ROSscavenging systems are essential for plants to cope with HT stress ${ }^{4,9,40}$. For instance, mutants lacking antioxidant pathways involved in ROS metabolism have been shown to be defective in basal thermotolerance ${ }^{4}$. In addition, overexpression of the apple ATG-encoding gene MdATG18a improved basal thermotolerance by enhancing antioxidant activity and reducing ROS accumulation $^{9}$. In this study, we examined ROS regulatory systems in WT plants and $M d V Q 37$ transgenic lines and found that the activities of CAT, POD, SOD, APX, GR, DHAR, and MDHAR in MdVQ37 transgenic lines were significantly lower than those in WT plants under HT stress (Figs. 2, 3). Consistent with the changes in the activities of ROS-scavenging enzymes, the total GSH and AsA levels, as well as the ratios of GSH/GSSG and AsA/DHA in the transgenic lines, were also significantly lower in the $M d V Q 37$ transgenic lines than in the WT plants under HT stress (Fig. 3). Therefore, both nonenzymatic and enzymatic ROS-scavenging regulatory systems were less active in the $M d V Q 37$ transgenic lines than in the WT plants under HT stress.

Several studies have also shown that a series of TFs, including HSFs, WRKYs, NACs, and bZIPs, are involved in the regulatory response of plants to HT stress. For example, HSFA4A overexpression enhances tolerance to HT stress by reducing oxidative damage ${ }^{41}$. hsfA3 mutant lines were shown to have reduced thermotolerance during the HT stress response ${ }^{13}$. Heat stress-induced WRKY39, a subgroup IId WRKY member, positively regulates the cooperation between the JA-activated and SA-activated signaling pathways, both of which mediate responses to HT stress in Arabidopsis $^{12}$. Functional analysis revealed that wrky 25 null mutants are sensitive to HT stress, while compared with WT Arabidopsis plants, transgenic plants overexpressing WRKY25 were relatively tolerant to HT stress, suggesting that WRKY25 is a positive regulator of thermotolerance ${ }^{42}$. Guan et al. ${ }^{14}$ reported that the NAC TF NAC019 can regulate the expression of heat stress-responsive genes. Transgenic plants overexpressing NAC019 have increased thermotolerance, and compared to WT plants, nac019 mutants have reduced tolerance to HT stress. In addition, overexpression of the tomato NAC TF SIJA2 reduced the content of endogenous SA, which led to significant changes in the physiology and gene expression patterns of transgenic tobacco plants, resulting in decreased heat tolerance of those plants ${ }^{43}$. In this study, the MdHSFA4A (MD09G1233700), MdHSFA3 (MD14G1015900), MdWRKY25 (MD11G1059400, MD12G1181000, and MD16G1066500), MdWRKY39 (MD07G1146900), and MdNAC019 (MD15G1136600 and MD15G1344900) genes, which are homologs of Arabidopsis HSFA4A, HSFA3, WRKY25, WRKY39, and NAC019, respectively, were significantly downregulated in the transgenic lines (Fig. 6, Table S2), suggesting that changes in the activities of many TFs in the transgenic lines negatively affected basal thermotolerance.

Previous studies have also shown that SA plays a key role in basal thermotolerance ${ }^{44,45}$. The SA-mediated signaling pathway has been shown to improve HT tolerance in Arabidopsis, cucumber, chickpea, grape, mustard, potato, tobacco, tomato, and wheat ${ }^{12,18,42,46-49}$. SA application can alleviate HT stress in grapes by inducing the activity of MDHAR, APX, and GR; increasing the redox ratio of AsA and GSH; and maintaining $\mathrm{Ca}^{2+}$ homeostasis under heat stress ${ }^{48}$. SA $(1.0 \mathrm{mM})$ was shown to increase proline production and restrict stress-induced ethylene formation after heat stress ${ }^{18}$. Treatment with $0.5 \mathrm{mM}$ SA was shown to decrease oxidative stress and EL and improve the maximum yield of PSII and $\mathrm{Fv} / \mathrm{Fm}$ in cucumber seedlings under HT stress ${ }^{47}$. Thus, appropriate concentrations of SA can improve heat stress by affecting the ROS-scavenging system, the AsA-GSH cycle, $\mathrm{Ca}^{2+}$ homeostasis, and the photosynthetic system. In addition, multiple studies have demonstrated the importance of endogenous SA and SA signaling pathways in basal thermotolerance. For instance, SA-deficient NahG transgenic Arabidopsis plants, which have decreased endogenous SA contents, present reduced basal thermotolerance, whereas SA-accumulating constitutive expressor of PR protein (cpr5) mutants, which have increased endogenous SA contents, exhibit an enhanced basal thermotolerant phenotype ${ }^{46,50}$. The Arabidopsis mutants npr1-1, npr1-5, and sid2, which are defective in SA signaling, present decreased tolerance to heat stress ${ }^{12,46,50,51}$. Thus, endogenous SA content and the SA-dependent signaling pathway play important roles in basal thermotolerance. In this study, overexpression of $M d V Q 37$ led to significant changes in the transcriptional activity of multiple TFs, which caused a significant increase in the transcript abundances of the possible target genes $M d S 5 H 1$ and $M d S 5 H 2$, resulting in significant accumulation of 2,5-DHBA in the transgenic lines (Figs. 6, 7). 2,5DHBA is one of the most widely produced aromatic acids in green plants and is suggested to be a component of the major pathway for SA catabolism ${ }^{38}$. The overaccumulation of 2,5-DHBA disrupted the homeostasis of endogenous SA in the transgenic lines. SA accumulation is essential for activating downstream gene expression of SA and SA-dependent resistance ${ }^{52}$. Indeed, in this study, under HT stress, the expression of the SA signaling- 


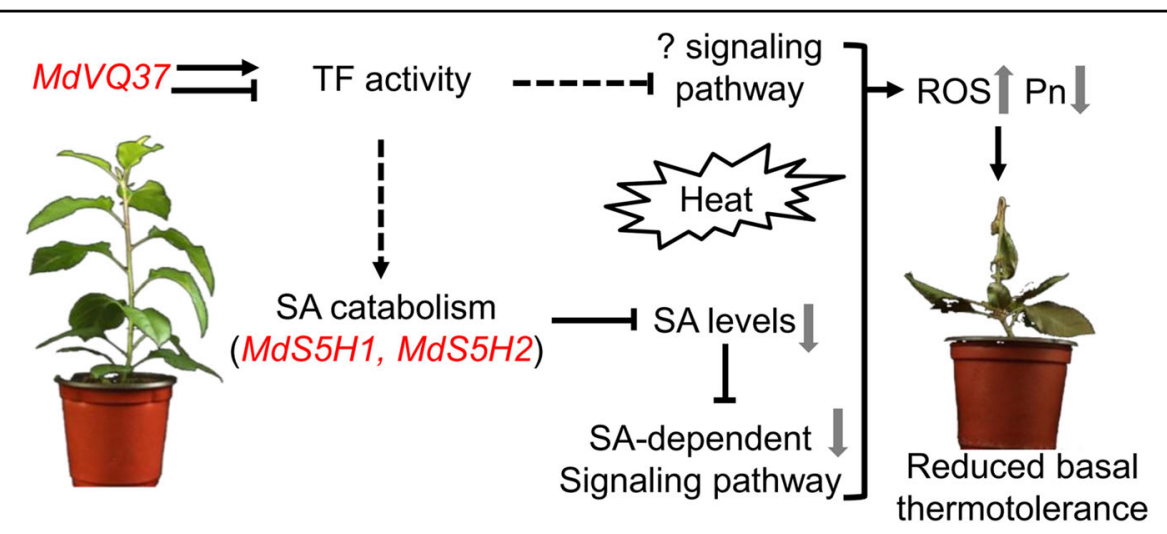

Fig. 8 Putative working mode of the MdVQ37 regulatory function in the apple response to HT stress. As a transcriptional regulator, overexpression of MdVQ37 can affect the transcriptional activity of multiple TFs, and indirectly up-regulated the expression of SA catabolism genes MdS5H1 and MdS5H2. This causes reduction in endogenous SA content and disruption in SA-dependent signaling pathways. Moreover, increased expression of MdVQ37 also resulted in unknown signal pathway. All of these changes resulted in higher ROS accumulation and lower photosynthetic capacity, and reduced basal thermotolerance under HT stress

related genes MdPAD4, MdPR3, and MdPR5 in the $M d V Q 37$ transgenic lines were significantly lower than that in the WT plants (Fig. 7C, D). In this case, the transgenic lines exhibited reduced activities of ROSscavenging enzymes (Figs. 2, 3), which attenuated AsAGSH recycling (Fig. 3) and photosynthetic capacity (Figs. $4,5)$ and ultimately led to reduced basal thermotolerance in the transgenic lines under HT stress. Moreover, spraying SA slightly increased the survival rate of the transgenic lines under HT stress (Fig. 7E), suggesting that the suppression of the SA level in the transgenic lines was partly responsible for the impaired basal thermotolerance. These findings also indicated that the impaired basal thermotolerance of the transgenic lines affected other signaling pathways.

In summary, we propose a putative working model of the regulatory function of MdVQ37 in the apple response to HT stress (Fig. 8). Overexpression of $M d V Q 37$, a transcriptional regulator, can affect the transcriptional activity of multiple TFs and indirectly upregulate the expression of the SA catabolism-related genes $M d S 5 H 1$ and $M d S 5 H 2$, causing a reduction in endogenous SA content and disruption of SA-dependent signaling pathways. Furthermore, the increased expression of $M d V Q 37$ triggered an as-yet-unknown signaling pathway. Together, all of these changes led to increased ROS accumulation, decreased photosynthetic capacity, and reduced basal thermotolerance under HT stress.

\section{Materials and methods}

\section{Plant materials and heat treatment}

GL-3 tissue culture-generated apple (Malus domestica 'Royal Gala') plants were used for genetic transformation to generate transgenic apple plants ${ }^{53}$. For expression analysis of $M d V Q$ genes, 45-day-old healthy GL-3 apple plants were maintained in a growth chamber at $48^{\circ} \mathrm{C}$. After heat exposure for 1,4 , or $8 \mathrm{~h}$, fully expanded apple leaves at the same position were collected for gene expression analysis of $M d V Q$ genes. For heat stress exposure, healthy, 90-day-old transgenic lines and WT plants of uniform size were placed in a growth chamber at $48^{\circ} \mathrm{C}$ for $2,4,6$, or $24 \mathrm{~h}$. In addition, transgenic lines and WT plants were maintained at room temperature $\left(24^{\circ} \mathrm{C}\right)$ as negative controls. After heat exposure for 2 and $4 \mathrm{~h}$, the 8th and 9th leaves from the bottom of the stem of six plants were collected for analysis of the expression of genes involved in SA biosynthesis, modification and catabolism, and signal transduction and for the determination of the SA content. After heat exposure for $6 \mathrm{~h}$, the 8th and 9th leaves from the bottom of the stem of six plants were harvested for damage assessment. All apple tissue materials were frozen immediately in liquid $\mathrm{N}_{2}$ and stored at $-80^{\circ} \mathrm{C}$. For survival rate analysis, healthy, 90-day-old plants of uniform size (heat exposure) were maintained in a growth chamber at $48^{\circ} \mathrm{C}$ for $24 \mathrm{~h}$, followed by a $48-\mathrm{h}$ recovery period at $24^{\circ} \mathrm{C}$. A plant was considered dead if $90 \%$ of its leaves failed to recover, and the plant survival ratio was determined (Fig. 1F). In addition, 90-day-old, healthy transgenic lines (SA + heat treatment) were sprayed with $100 \mu \mathrm{M}$ SA solution, and control plants were sprayed with water. At $24 \mathrm{~h}$ after pretreatment, the plants were maintained in a growth chamber at $48^{\circ} \mathrm{C}$ for $24 \mathrm{~h}$, followed by a 48 -h recovery period at $24^{\circ} \mathrm{C}$, and the plant survival ratio was determined (Fig. 7F).

\section{Construction of plasmids and genetic transformation of apple}

We used RT-PCR to isolate the coding region of $M d V Q 37$ from mature leaves of Royal Gala apple plants to construct an $M d V Q 37$ overexpression vector ${ }^{31}$. The 
coding region was inserted into a pCAMBIA2300 plant transformation vector driven by the cauliflower mosaic virus (CaMV) $35 \mathrm{~S}$ promoter. Agrobacterium-mediated genetic transformation of apple leaves was performed with GL-3 as the genetic background and EHA105 as the infectious strain, as described previously ${ }^{53}$. Regenerated buds were screened with $25 \mathrm{mg} / \mathrm{L}$ kanamycin monosulfate (Kan). Kan-resistant seedlings were subcultured every month in subculture media. The transgenic lines were then confirmed via genomic PCR amplification analysis (Table S5). The overexpression of $M d V Q 37$ was evaluated via qRT-PCR analysis (Table S6).

\section{Evaluation of stress tolerance}

The EL of the apple leaves was analyzed as previously described $^{54,55}$. The levels of proline and MDA were measured using PRO-2-Y and MDA-2-Y kits (Comin, Suzhou, China), respectively, according to the manufacturer's instructions. The chlorophyll concentrations were determined according to a previously described method ${ }^{56}$.

\section{Antioxidant enzyme activities and histochemical staining}

The levels of oxidized glutathione (GSSG), GSH, AsA, DHA, $\mathrm{H}_{2} \mathrm{O}_{2}$, and $\mathrm{O}_{2}$.- and the activities of CAT, POD, SOD, GR, DHAR, APX, and MDHAR were analyzed using the following test kits purchased from Comin: GSSG-2W, GSH-2-W, AsA-2A-W, DHA-2-W, $\mathrm{H}_{2} \mathrm{O}_{2}-2-\mathrm{Y}, \mathrm{SA}-2-$ G, CAT-2-Y, POD-2-Y, SOD-2-Y, APX-2-W, GR-2-W, MDHAR-2-W, and DHAR-2-W, respectively. The manufacturer's instructions were followed for each kit. After $6 \mathrm{~h}$ of heat exposure, fresh solutions of NBT and DAB were used to stain the leaf samples with $\mathrm{O}_{2}--$ and $\mathrm{H}_{2} \mathrm{O}_{2}$, respectively. The stained leaves were immersed in a $70 \%$ ethanol decolorizing solution at $95^{\circ} \mathrm{C}$ in a water bath for $4 \mathrm{~h}$ and then transferred to a $10 \%$ glycerin solution for observations and imaging.

\section{Evaluation of photosynthetic characteristics and chlorophyll fluorescence}

After a 24-h recovery period following $6 \mathrm{~h}$ of heat exposure, leaf $\mathrm{Pn}, \mathrm{Tr}, \mathrm{Ci}$, and $\mathrm{g}_{\mathrm{s}}$ were measured using a CIRAS-3 portable photosynthesis system (PP System, Amesbury, MA, USA) ${ }^{9}$. Transient chlorophyll fluorescence was examined using an Open FluorCam FC 800-O instrument, and $\mathrm{Fv} / \mathrm{Fm}$ ratios were calculated using Fluorcam7 software (PSI, Brno, Czech Republic) ${ }^{9,57}$. For all heat exposure experiments, photosynthetic characteristics and chlorophyll fluorescence were measured on the 6th and 7th leaves from the bottom of the stems of each selected plant.

\section{Measurement of stomatal aperture}

After $4 \mathrm{~h}$ of heat exposure, fully expanded leaves were removed from the same position of each selected plant and immediately cut into small square pieces $(0.5 \times$ $0.5 \mathrm{~cm})$. The samples were subsequently immersed in a $4 \%$ glutaraldehyde solution to avoid any alteration or damage during sample preparation and maintained at $4{ }^{\circ} \mathrm{C}$ for $24 \mathrm{~h}$. The stomata were then observed via scanning electron microscopy (SEM) with a JSM-6360LV scanning electron microscope (JEOL, Ltd., Tokyo, Japan), as previously described ${ }^{56}$.

\section{Measurements of energy conversion and electron transport in PSI and PSII}

After a 24-h recovery period, following $6 \mathrm{~h}$ of heat exposure, the PSI [Y(I) and ETR(I)], and PSII [Y(II) and ETR(II)] activities of the apple leaves were measured simultaneously using a Dual-PAM-100 system (Heinz Walz GmbH, Effeltrich, Germany) in conjunction with a Fluo C P700 instrument as previously described ${ }^{9,57}$.

\section{RNA preparation, transcriptome sequencing, and differentially expressed gene analysis}

For transcriptome analysis, 90-day-old leaves (eighth leaf) from the bottom of the stem of healthy plants were collected for RNA extraction. Total RNA from three groups of leaves (WT, 37-1, and 37-2) was isolated using a DP441-RNA Prep Pure Plant Plus Kit (Tiangen, Beijing, China) according to the manufacturer's instructions. Illumina RNA sequencing was then performed by Novogene Bioinformatics Technology Co., Ltd. (Beijing, China) on an Illumina HiSeq 4000 platform, following previously described methods ${ }^{58}$. DEGs with a fold change $\geq 2$ and an FDR $\leq 0.05$ were identified using the DESeq2 $\mathrm{R}$ package $(1.16 .1)^{59}$. KEGG pathway enrichment analysis and GO enrichment analysis of the DEGs were performed using cluster Profiler (3.4.4) software $^{60}$.

\section{RNA isolation and qRT-PCR analysis}

Total RNA from apple leaves was isolated using a Wolact Plant RNA Isolation Kit (Wolact, Hong Kong, China). cDNA was synthesized using a PrimeScript FirstStrand cDNA Synthesis Kit (TaKaRa, Dalian, China). qRT-PCR was performed on a LightCycler® 96 quantitative instrument (Roche Diagnostics, Basel, Switzerland) in conjunction with TransStart Top Green qRT-PCR SuperMix (Transgen Biotech, Beijing, China), as described previously $^{31}$. Three biological replications were used for each assay, and the $M d M D H$ gene was used as an endogenous control to normalize the transcript levels of different genes (Table S6 ${ }^{61}$. The gene expression was determined using the $2^{-\Delta \Delta C t}$ method $^{62}$.

\section{Metabolite extraction}

SA was extracted and purified according to previously described methods ${ }^{63,64}$. Briefly, after accurately weighing $0.1 \mathrm{~g}$ of apple leaves, the leaves were ground in liquid 
nitrogen, suspended in $1 \mathrm{~mL}$ of methanol, and incubated at $-20{ }^{\circ} \mathrm{C}$ overnight. Subsequently, the suspension was centrifuged at $13,000 \mathrm{rpm}$ for $15 \mathrm{~min}$ at $4{ }^{\circ} \mathrm{C}$, the supernatant was air-dried with nitrogen gas, and the extract was dissolved in $200 \mu \mathrm{L}$ of $80 \%$ methanol. Then, a $100-\mu \mathrm{L}$ aliquot of the extract was air-dried under a stream of nitrogen gas and resuspended in $500 \mu \mathrm{L}$ of sodium acetate buffer (0.1 M; pH 5.5). A $200 \mu \mathrm{L}$ aliquot of the suspension was used to analyze the free SA content via liquid chromatography-mass spectrometry (LC-MS). In addition, a $200-\mu \mathrm{L}$ aliquot of the suspension was treated with $10 \mu \mathrm{L}$ of $\beta$-glucosidase, incubated in a water bath at $37^{\circ} \mathrm{C}$ for $2 \mathrm{~h}$, and then boiled in water for $5 \mathrm{~min}$ to stop the enzymatic reaction. Afterward, the suspension was centrifuged at $13,000 \times g$ for $10 \mathrm{~min}$ at $4{ }^{\circ} \mathrm{C}$, after which the supernatant was subjected to LC-MS analysis to determine the total SA content. 2,5-DHBA was extracted according to previously described methods ${ }^{65}$. Briefly, after accurately weighing $0.1 \mathrm{~g}$ of apple leaves, the leaves were ground in liquid nitrogen, suspended in $1 \mathrm{~mL}$ of extraction solution (methanol:water:formic acid (25:24:1)), subjected to ultrasonic treatment for $20 \mathrm{~min}$ (normal temperature, $40 \mathrm{~Hz}, 100 \mathrm{~W}$ ), centrifuged at $12,000 \mathrm{rpm}$ for $15 \mathrm{~min}$ at $4{ }^{\circ} \mathrm{C}$, and then filtered through a $0.25 \mu \mathrm{m}$ filter membrane. The filtrate was ultimately subjected to LCMS analysis to determine the 2,5-DHBA concentration.

\section{Liquid chromatography-mass spectrometry (LC-MS) analysis}

The LC-MS technique was used as described previously $^{64}$. A QTRAP ${ }^{\circledR} 5500$ LC-MS/MS system (Applied Biosystems/MDS Analytical Technologies, Foster City, California, USA) equipped with an InertSustain AQ-C18 column $(4.6 \times 150 \mathrm{~mm}, 5 \mathrm{~mm}$; GL Sciences, Torrance, CA, USA) was used to analyze the metabolites. The mobile phase consisted of $0.1 \%(\mathrm{v} / \mathrm{v})$ formic acid (A) and methanol (B). To measure the SA content, a gradient elution process was implemented through the following steps: $75 \%$ A ( $0 \mathrm{~min}), 75 \%$ A ( $1 \mathrm{~min}), 5 \%$ A ( $5 \mathrm{~min}), 5 \%$ A (6.5 $\mathrm{min}), 75 \% \mathrm{~A}(6.6 \mathrm{~min})$, and $75 \% \mathrm{~A}(13 \mathrm{~min})$. For the measurement of the 2,5-DHBA content, the gradient elution process was implemented as follows: $75 \% \mathrm{~A}$ (0 min), 65\% A (1 min), 5\% A (6 min), 5\% A (8.9 min), and $75 \% \mathrm{~A}(9 \mathrm{~min})$. The flow rate of the aliquot was maintained at $0.7 \mathrm{~mL} \mathrm{~min}^{-1}$ throughout the process. The concentrations of free SA, total SA, and 2,5-DHBA were analyzed by calculating the LC-MS peak area based on standard curves.

\section{Statistical analysis}

All the data were obtained from three biological replications and analyzed with IBM SPSS (version 20) statistical software (IBM Corporation, Armonk, NY, USA). The experimental data are expressed as the mean values \pm standard errors (SEs). One-way analysis of variance (ANOVA) was performed to compare significant differences between the WT plants and transgenic lines based on Duncan's test $(p<0.05)$.

\section{Acknowledgements}

This work was supported by the National Key Research and Development Program of China (2018YFD1000303), earmarked funds for the China Agriculture Research System (CARS-27), and the National Natural Science Foundation of China (31401852 and 31701894). The authors are grateful to Dr. Zhihong Zhang for providing tissue culture-generated GL-3 apple plants.

\section{Author contributions}

F.M., K.M., and Q.D. conceived and designed the experiments. Q.D., D.D., W.Z. D.H., Q.W., and X.L. performed the experiments. F.M., K.M., and Q.D. analyzed the data. F.M. and Q.D. wrote the manuscript. All the authors have read and approved the manuscript.

\section{Competing interests}

The authors declare no competing interests.

Supplementary information The online version contains supplementary material available at https://doi.org/10.1038/s41438-021-00655-3.

Received: 17 March 2021 Revised: 9 June 2021 Accepted: 18 June 2021 Published online: 01 October 2021

\section{References}

1. Fedoroff, N. V. et al. Radically rethinking agriculture for the 21st century. Science 327, 833-834 (2010).

2. Zhu, J. K. Abiotic stress signaling and responses in plants. Cell 167, 313-324 (2016).

3. Sung, D., Kaplan, F., Lee, K. J. \& Guy, C. L. Acquired tolerance to temperature extremes. Trends Plant Sci. 8, 179-187 (2003).

4. Larkindale, J., Hall, J. D., Knight, M. R. \& Vierling, E. Heat stress phenotypes of Arabidopsis mutants implicate multiple signaling pathways in the acquisition of thermotolerance. Plant Physiol. 138, 882-897 (2005).

5. Suzuki, N., Bajad, S., Shuman, J., Shulaev, V. \& Mittler, R. The transcriptional coactivator MBFIC is a key regulator of thermotolerance in Arabidopsis thaliana. J. Biol. Chem. 283, 9269-9275 (2008).

6. Hasanuzzaman, M., Nahar, K, Alam, M. M., Roychowdhury, R. \& Fujta, M. Physiological, biochemical, and molecular mechanisms of heat stress tolerance in plants. Int. J. Mol. Sci. 14, 9643-9684 (2013).

7. De Ronde, J. A. et al. Photosynthetic response of transgenic soybean plants, containing an Arabidopsis P5CR gene, during heat and drought stress. J. Plant Physiol. 161, 1211-1224 (2004).

8. Kepova, K. D., Holzer, R., Stoilova, L. S. \& Feller, U. Heat stress effects on ribulose1,5-bisphosphate carboxylase/oxygenase, rubisco binding protein and rubisco activase in wheat leaves. Biol. Plant. 49, 521-525 (2005).

9. Huo, L. et al. MdATG18a overexpression improves basal thermotolerance in transgenic apple by decreasing damage to chloroplasts. Hortic. Res. 7, 21 (2020).

10. Ohama, N., Sato, H., Shinozaki, K. \& Yamaguchi-Shinozaki, K. Transcriptional regulatory network of plant heat stress response. Trends Plant Sci. 22, 53-65 (2017).

11. Li, C. et al. Dopamine alleviates salt-induced stress in malus hupehensis. Physiol. Plant. 153, 584-602 (2015).

12. Li, S., Zhou, X., Chen, L., Huang, W. \& Yu, D. Functional characterization of Arabidopsis thaliana WRKY39 in heat stress. Mol. Cells 29, 475-483 (2010).

13. Schramm, F. et al. A cascade of transcription factor DREB2A and heat stress transcription factor HsfA3 regulates the heat stress response of Arabidopsis. Plant J. 53, 264-274 (2008).

14. Guan, Q., Yue, X., Zeng, H. \& Zhu, J. The protein phosphatase RCF2 and its interacting partner NAC019 are critical for heat stress-responsive gene regulation and thermotolerance in Arabidopsis. Plant Cell. 26, 438-453 (2014). 
15. Liao, C., Zheng, Y. \& Guo, Y. MYB30 transcription factor regulates oxidative and heat stress responses through ANNEXIN-mediated cytosolic calcium signaling in Arabidopsis. N. Phytol. 216, 163-177 (2017).

16. Gao, H., Brandizzi, F., Benning, C. \& Larkin, R. M. A membrane-tethered transcription factor defines a branch of the heat stress response in Arabidopsis thaliana. Proc. Natl Acad. Sci. USA 105, 16398-16403 (2008).

17. Miura, K. \& Tada, Y. Regulation of water, salinity, and cold stress responses by salicylic acid. Front. Plant Sci. 5, 4 (2014).

18. Khan, M. I. R., Iqbal, N., Masood, A., Per, T. S. \& Khan, N. A. Salicylic acid alleviates adverse effects of heat stress on photosynthesis through changes in proline production and ethylene formation. Plant Signal. Behav. 8, e26374 (2013).

19. Zandalinas, S. I., Rivero, R. M., Martinez, V., Gomez-Cadenas, A. \& Arbona, $V$. Tolerance of citrus plants to the combination of high temperatures and drought is associated to the increase in transpiration modulated by a reduction in abscisic acid levels. BMC Plant Biol. 16, 105 (2016).

20. Kamran, M. et al. Modulation of growth performance and coordinated induction of ascorbate-glutathione and methylglyoxal detoxification systems by salicylic acid mitigates salt toxicity in choysum (Brassica parachinensis L.). Ecotoxicol. Environ. Saf. 188, 109877 (2020).

21. Li, Q. et al. Enhancement of endogenous SA accumulation improves poornutrition stress tolerance in transgenic tobacco plants overexpressing a SAbinding protein gene. Plant Sci. 292, 110384 (2020).

22. Morikawa, K., Shiina, T., Murakami, S. \& Toyoshima, Y. Novel nuclear-encoded proteins interacting with a plastid sigma factor, Sig1, in Arabidopsis thaliana. FEBS Lett. 514, 300-304 (2002).

23. Jing, Y. \& Lin, R. The VQ motif-containing protein family of plant-specific transcriptional regulators. Plant Physiol. 169, 371-378 (2015).

24. Wang, A. et al. The VQ motif protein IKU1 regulates endosperm growth and seed size in Arabidopsis. Plant J. 63, 670-679 (2010).

25. Pan, J., Wang, H., Hu, Y. \& Yu, D. Arabidopsis VQ18 and VQ26 proteins interact with $\mathrm{ABI} 5$ transcription factor to negatively modulate $\mathrm{ABA}$ response during seed germination. Plant J. 95, 529-544 (2018).

26. Li, Y., Jing, Y., Li, J., Xu, G. \& Lin, R. Arabidopsis VQ MOTIF-CONTAINING PROTEIN29 represses seedling deetiolation by interacting with PHYTOCHROMEINTERACTING FACTOR1. Plant Physiol. 164, 2068-2080 (2014).

27. Lei, R. et al. Arabidopsis WRKY2 and WRKY34 transcription factors interact with VQ20 protein to modulate pollen development and function. Plant J. 91, 962-976 (2017).

28. Wang, H., Hu, Y., Pan, J. \& Yu, D. Arabidopsis VQ motif-containing proteins VQ12 and VQ29 negatively modulate basal defense against Botrytis cinerea. Sci. Rep. 5, 14185 (2015).

29. $\mathrm{Hu}, \mathrm{Y}$. et al. Arabidopsis transcription factor WRKY8 functions antagonistically with its interacting partner VQ9 to modulate salinity stress tolerance. Plant J. 74, 730-745 (2013).

30. Ding, $\mathrm{H}$. et al. Genome-wide analysis of the plant-specific VQ motif-containing proteins in tomato (Solanum lycopersicum) and characterization of SIVQ6 in thermotolerance. Plant Physiol. Bioch. 143, 29-39 (2019).

31. Dong, Q. et al. Structural and functional analyses of genes encoding VQ proteins in apple. Plant Sci. 272, 208-219 (2018).

32. Mittler, R. et al. ROS signaling: the new wave? Trends Plant Sci. 16, 300-309 (2011).

33. Gill, S. S. \& Tuteja, N. Reactive oxygen species and antioxidant machinery in abiotic stress tolerance in crop plants. Plant Physiol. Biochem. 48, 909-930 (2010).

34. Wang, P. et al. Delayed senescence of apple leaves by exogenous melatonin treatment: toward regulating the ascorbate-glutathione cycle. J. Pineal Res. 53, $11-20$ (2012).

35. Wang, X., Xu, C., Cai, X., Wang, Q. \& Dai, S. Heat-responsive photosynthetic and signaling pathways in plants: insight from proteomics. Int. J. Mol. Sci. 18, 2191 (2017).

36. Zhou, J., Wang, J., Yu, J. Q. \& Chen, Z. Role and regulation of autophagy in heat stress responses of tomato plants. Front Plant Sci. 5, 174 (2014).

37. Porcar-Castell, A. et al. Linking chlorophyll a fluorescence to photosynthesis for remote sensing applications: mechanisms and challenges. J. Exp. Bot. 65 4065-4095 (2014)

38. Zhang, Y. et al. S5H/DMR6 encodes a salicylic acid 5-hydroxylase that fine-tunes salicylic acid homeostasis. Plant Physiol. 175, 1082-1093 (2017).
39. Yamasaki, K., Kigawa, T., Seki, M., Shinozaki, K. \& Yokoyama, S. DNA binding domains of plant-specific transcription factors: structure, function, and evolution. Trends Plant Sci. 18, 267-276 (2013).

40. de Pinto, M. C., Locato, V., Paradiso, A. \& De Gara, L. Role of redox homeostasis in thermo-tolerance under a climate change scenario. Ann. Bot. 116, 487-496 (2015).

41. Andrási, N. et al. The mitogen-activated protein kinase 4-phosphorylated heat shock factor A4A regulates responses to combined salt and heat stresses. J. Exp. Bot. 70, 4903-4918 (2019).

42. Li, S. J., Fu, Q. T., Huang, W. D. \& Yu, D. Q. Functional analysis of an Arabidopsis transcription factor WRKY25 in heat stress. Plant Cell Rep. 28, 683-693 (2009).

43. Liu, Z. M. et al. Over-expression of SIJA2 decreased heat tolerance of transgenic tobacco plants via salicylic acid pathway. Plant Cell Rep. 36 529-542 (2017).

44. Khan, M. I., Fatma, M., Per, T. S., Anjum, N. A. \& Khan, N. A. Salicylic acid-induced abiotic stress tolerance and underlying mechanisms in plants. Front. Plant Sci. 6, 462 (2015)

45. Herrera-Vásquez, A., Salinas, P. \& Holuigue, L. Salicylic acid and reactive oxygen species interplay in the transcriptional control of defense genes expression. Front. Plant Sci. 6, 171 (2015).

46. Clarke, S. M., Mur, L. A., Wood, J. E. \& Scott, I. M. Salicylic acid dependent signaling promotes basal thermotolerance but is not essential for acquired thermotolerance in Arabidopsis thaliana. Plant J. 38, 432-447 (2004).

47. Shi, Q., Bao, Z., Zhu, Z., Ying, Q. \& Qian, Q. Effects of different treatments of salicylic acid on heat tolerance, chlorophyll fluorescence, and antioxidant enzyme activity in seedlings of Cucumis sativa L. Plant Growth Regul. 48, 127-135 (2006)

48. Wang, L. J. \& Li, S. H. Salicylic acid-induced heat or cold tolerance in relation to $\mathrm{Ca}^{2+}$ homeostasis and antioxidant systems in young grape plants. Plant Sci. 170, 685-694 (2006)

49. Zhao, H. J. et al. Effects of salicylic acid on protein kinase activity and chloroplast D1 protein degradation in wheat leaves subjected to heat and high light stress. Acta Ecologica Sin. 31, 259-263 (2011).

50. Clarke, S. M. et al. Jasmonates act with salicylic acid to confer basal thermotolerance in Arabidopsis thaliana. N. Phytol. 182, 175-187 (2009).

51. Ahammed, G. J., Li, X., Yu, J. \& Shi, K. NPR1-dependent salicylic acid signaling is not involved in elevated $\mathrm{CO}_{2}$-induced heat stress tolerance in Arabidopsis thaliana. Plant Signal. Behav. 10, e1011944 (2015).

52. Shi, H. et al. The cysteine2/histidine2-type transcription factor ZINC FINGER OF ARABIDOPSIS THALIANA6 modulates biotic and abiotic stress responses by activating salicylic acid-related genes and C-REPEAT-BINDING FACTOR genes in Arabidopsis. Plant Physiol. 165, 1367-1379 (2014).

53. Dai, $\mathrm{H}$. et al. Development of a seedling clone with high regeneration capacity and susceptibility to Agrobacterium in apple. Sci. Hortic. 164, 202-208 (2013).

54. Dionisio-Sese, M. L. \& Tobita, S. Antioxidant responses of rice seedings to salinity stress. Plant Sci. 135, 1-9 (1998).

55. Sun, X. et al. Improvement of drought tolerance by overexpressing MdATG18a is mediated by modified antioxidant system and activated autophagy in transgenic apple. Plant Biotechnol. J. 16, 545-557 (2018).

56. Liang, B. et al. Effects of exogenous dopamine on the uptake, transport, and resorption of apple ionome under moderate drought. Front. Plant Sci. 9, 14 (2018).

57. Deng, C., Zhang, D., Pan, X., Chang, F. \& Wang, S. Toxic effects of mercury on PSI and PSII activities, membrane potential and transthylakoid proton gradient in Microsorium pteropus. J. Photochem. Photobiol. B. Biol. 127, 1-7 (2013).

58. Zhang, R. P. et al. Transcriptional profling identifies location-specifc and breedspecifc differentially expressed genes in embryonic myogenesis in Anas platyrhynchos. PLOS ONE 10, e0143378 (2015)

59. Love, M. I., Huber, W. \& Anders, S. Moderated estimation of fold change and dispersion for RNA-seq data with DESeq2. Genome Biol. 15, 550 (2014)

60. Yu, G., Wang, L. G., Han, Y. \& He, Q. Y. clusterProfiler: an R package for comparing biological themes among gene clusters. OMICS 16, 284-287 (2012).

61. Perini, P., Pasquali, G., Margis-Pinheiro, M., de Oliviera, P. R. D. \& Revers, L. F. Reference genes for transcriptional analysis of flowering and fruit ripening stages in apple (Malus $\times$ domestica Borkh.). Mol. Breed. 34, 829-842 (2014) 
62. Livak, K. J. \& Schmittgen, T. D. Analysis of relative gene expression data using real-time quantitative PCR and the 2- $\triangle \triangle C T$ method. Methods 25, 402-408 (2001).

63. Fu, J., Chu, J., Sun, X., Wang, J. \& Yan, C. Simple, rapid, and simultaneous assay of multiple carboxyl containing phytohormones in wounded tomatoes by UPLC-MS/MS using single SPE purification and isotope dilution. Anal. Sci. 28, 1081-1087 (2012).
64. Zhou, K. et al. MdUGT88F1-mediated phloridzin biosynthesis regulates apple development and Valsa canker resistance. Plant Physiol. 180, 2290-2305 (2019).

65. Zhang, Y. et al. Developmental changes of carbohydrates, organic acids, amino acids, and phenolic compounds in 'Honeycrisp' apple flesh. Food Chem. 123, 1013-1018 (2010). 